Homology, Homotopy and Applications, vol. 20(2), 2018, pp.61-77

\title{
GENERALIZED STEENROD HOMOLOGY THEORIES ARE IDENTICAL WITH PARTIALLY CONTINUOUS HOMOLOGY THEORIES
}

\author{
PETER MROZIK \\ (communicated by George Janelidze)
}

\begin{abstract}
It is shown that generalized Steenrod homology theories are identical with partially continuous homology theories which are characterized by a short exact $\lim ^{1}$-sequence.
\end{abstract}

\section{Introduction}

In [22] Milnor gave an axiomatic characterization of classic Steenrod homology as an ordinary homology theory on the category $\mathbf{C M}^{\mathbf{2}}$ of compact metric pairs which in addition satisfies the strong excision axiom and the cluster axiom. An important feature of Steenrod homology is that for any $(X, A) \in \mathbf{C M}^{2}$ and any inverse sequence $(\mathbf{X}, \mathbf{A})$ in $\mathbf{C M}^{\mathbf{2}}$ such that $(X, A)=\lim (\mathbf{X}, \mathbf{A})$ one obtains a short exact sequence (cf. $[\mathbf{2 2}$, Theorem 4])

$$
0 \rightarrow \lim ^{1} h_{n+1}(\mathbf{X}, \mathbf{A}) \stackrel{\beta}{\rightarrow} h_{n}(X, A) \stackrel{\gamma}{\rightarrow} \underset{\longleftarrow}{\lim } h_{n}(\mathbf{X}, \mathbf{A}) \rightarrow 0 .
$$

There are two obvious ways of generalizing Milnor's axiomatic approach to Steenrod homology: (1) Enlarging the domain on which ordinary homology theories are defined and (2) dropping the dimension axiom. Both are success stories.

(1) Milnor's characterization of Steenrod homology has been considerably extended by Berikashvili $[\mathbf{2}, \mathbf{3}, 4]$, Inassaridze $[\mathbf{1 1}, \mathbf{1 2}]$ and Mdzinarishvili $[\mathbf{1 2}, \mathbf{1 8}, \mathbf{1 9}, \mathbf{2 0}, 21]$ who specified various collections $\mathfrak{A}$ of axioms assuring the uniqueness of ordinary homology theories on the category $\mathbf{C o m}^{2}$ of compact Hausdorff pairs which satisfy $\mathfrak{A}$. See $[\mathbf{2 0}, \mathbf{2 1}]$ for an overview. Most of the concrete ordinary homology theories on $\mathbf{C o m}^{2}$ constructed in the literature satisfy the Berikashvili axioms so that they are essentially identical. The unique theory satisfying the Berikashvili axioms is called the ordinary Steenrod homology theory on $\mathbf{C o m}^{2}$. An elegant characterization of this homology theory is based on the axiom of partial continuity introduced by Inassaridze and Mdzinarishvili $[\mathbf{1 1}, \mathbf{1 2}]$. This axiom says that for any $(X, A) \in \mathbf{C o m}^{\mathbf{2}}$ and any inverse system $(\mathbf{X}, \mathbf{A})$ of compact polyhedral pairs such that $(X, A)=\lim _{\longleftarrow}(\mathbf{X}, \mathbf{A})$

Received April 22, 2016, revised December 17, 2016; published on May 2, 2018. 2010 Mathematics Subject Classification: 55N40, 55N20, 55P55.

Key words and phrases: Steenrod homology theory, partially continuous homology theory. Article available at http://dx.doi.org/10.4310/HHA.2018.v20.n2.a4

Copyright (C) 2018, Peter Mrozik. Permission to copy for private use granted. 
there exists a short exact sequence

$$
0 \rightarrow \lim ^{1} h_{n+1}(\mathbf{X}, \mathbf{A}) \stackrel{\beta}{\rightarrow} h_{n}(X, A) \stackrel{\mathbf{p}_{\#}}{\rightarrow} \lim _{\longleftarrow} h_{n}(\mathbf{X}, \mathbf{A}) \rightarrow 0,
$$

in which $\mathbf{p}:(X, A) \rightarrow(\mathbf{X}, \mathbf{A})$ is the canonical inverse limit morphism and $\beta$ is natural with respect to level morphisms between inverse systems. This is a variation of Milnor's $\lim ^{1}$-sequence which is far more subtle than a straightforward transfer from $\mathbf{C M}^{2}$ to $\mathbf{C o m}^{2}$. On the one hand it is more special since it only deals with inverse systems of compact polyhedral pairs. On the other hand it is more precise because it involves the canonical homomorphism $\mathbf{p}_{\#}$ and requires $\beta$ to be natural in a specific sense.

(2) Dropping the dimension axiom leads to the concept of generalized Steenrod homology theories. Originally they were introduced in [14] by Kaminker and Schochet as single space theories for unpointed compact metric spaces (concerning unpointed single space theories see Kelly [15]). In the subsequent paper [13] the perspective was changed by defining them as single space theories for pointed compact metric spaces. See Section 2 for details.

For generalized homology theories it does not make sense to search for uniqueness theorems. Instead it is of interest to clarify the status of additional axioms imposed on such theories. Focusing on generalized homology theories for compact metric spaces, we proved in [23] that the strong excision axiom is equivalent to strong shape invariance. In this paper we continue this program by showing that the strong excision axiom plus the cluster axiom are equivalent to an adequate version of the axiom of partial continuity.

\section{Preliminaries}

Let $\mathbf{C M}, \mathbf{C M}_{\mathbf{0}}$ and $\mathbf{C M}^{\mathbf{2}}$ denote the categories of compacta (= compact metric spaces), pointed compacta and pairs of compacta, respectively. We regard CM and $\mathbf{C M}_{\mathbf{0}}$ as full subcategories of $\mathbf{C M}^{\mathbf{2}}$ by identifying $X \in \mathbf{C M}$ with $(X, \emptyset)$ and $\left(X, x_{0}\right) \in$ $\mathbf{C M}_{\mathbf{0}}$ with $\left(X,\left\{x_{0}\right\}\right)$. Let $\mathbf{C M}_{\mathbf{n e}}$ denote the category of nonempty compacta.

A reduced homology system $[\mathbf{2 3}]$ on one of the categories $\mathbf{C}=\mathbf{C M}_{\mathbf{n e}}, \mathbf{C M}_{\mathbf{0}}$ is a system $\left(h_{n}, \sigma_{n}\right)_{n \in \mathbb{Z}}$ of covariant homotopy invariant functors $h_{n}: \mathbf{C} \rightarrow \mathbf{A b}$ (= category of abelian groups) and of natural isomorphisms $\sigma_{n}: h_{n} \rightarrow h_{n+1} \circ \mathbf{S}$, where $\mathbf{S}$ denotes the suspension functor on $\mathbf{C}$ (i.e. unreduced suspension on $\mathbf{C M}_{\mathbf{n e}}$ and reduced suspension on $\mathbf{C M}_{\mathbf{0}}$ ). A reduced homology system is called exact resp. quotient exact if for each pair $(X, A)$ with components in $\mathbf{C}$ the natural sequence

$$
h_{n}(A) \stackrel{i_{*}}{\rightarrow} h_{n}(X) \stackrel{j_{*}}{\rightarrow} h_{n}(X \cup C A) \text { resp. } h_{n}(A) \stackrel{i_{*}}{\rightarrow} h_{n}(X) \stackrel{p_{*}}{\rightarrow} h_{n}(X / A)
$$

is exact for all $n \in \mathbb{Z}$. Here, $X \cup C A$ denotes the adjunction space obtained by attaching the cone $C A$ to $X$ via the inclusion $A \hookrightarrow C A$ ( $C A$ denotes the unreduced cone $\mathbf{C M}_{\text {ne }}$ and the reduced cone on $\mathbf{C M}_{\mathbf{0}}$. In the pointed case $X \cup C A$ and $X / A$ are understood as pointed spaces in the obvious way).

Exact reduced homology systems are customarily denoted as reduced homology theories. Since each quotient exact reduced homology system is a reduced homology theory (cf. [23]), we also use the phrase quotient exact reduced homology theory. 
An (unreduced) homology theory on $\mathbf{C M}^{2}$ is a system $\left(h_{n}, \partial_{n}\right)_{n \in \mathbb{Z}}$ of covariant homotopy invariant functors $h_{n}: \mathbf{C M}^{2} \rightarrow \mathbf{A b}$ and of natural transformations $\partial_{n}: h_{n+1}(X, A) \rightarrow h_{n}(A, \emptyset)=h_{n}(A)$ such that the exactness axiom and the excision axiom are satisfied. If the $h_{n}$ satisfy the strong excision axiom:

(SE) For each compact metric pair $(X, A)$, the quotient map $p:(X, A) \rightarrow(X / A, *)$ induces isomorphisms $p_{*}: h_{n}(X, A) \rightarrow h_{n}(X / A, *)$ for all $n \in \mathbb{Z}$

we call $\left(h_{n}, \partial_{n}\right)$ a strongly excisive homology theory. Note that we set $(X / \emptyset, *)=$ $\left(X^{+}, *\right)$ where $X^{+}$is the disjoint union of $X$ and a single point space $*$.

Definition 2.1. Let $\mathfrak{h}$ be a homology theory on $\mathbf{C}=\mathbf{C M}_{\mathbf{n e}}, \mathbf{C M}_{\mathbf{0}}, \mathbf{C M}^{2}$.

1. $\mathfrak{h}$ is said to be of strong type if it is quotient exact for $\mathbf{C}=\mathbf{C M}_{\mathbf{n e}}, \mathbf{C M}_{\mathbf{0}}$ resp. strongly excisive for $\mathbf{C}=\mathbf{C M}^{2}$.

2. $\mathfrak{h}$ is called a Steenrod homology theory if it is of strong type and in addition satisfies the following cluster axiom:

(Cl) For each sequence $X_{i}=\left(X_{i}, x_{i}\right)$ of pointed compacta the projections

$$
\pi_{k}: \mathbf{C l}_{i=1}^{\infty}\left(X_{i}, x_{i}\right) \rightarrow X_{k}
$$

defined on the cluster $\mathbf{C l}_{i=1}^{\infty}\left(X_{i}, x_{i}\right)$ induce isomorphisms for all $n \in \mathbb{Z}$

$$
\Pi: h_{n}\left(\mathbf{C l}_{i=1}^{\infty}\left(X_{i}, x_{i}\right)\right) \rightarrow \prod_{k=1}^{\infty} h_{n}\left(X_{k}\right), \Pi(x)=\left(\left(\pi_{k}\right)_{*}(x)\right) \text {. }
$$

Domain and range of $\pi_{k}$ are regarded as unpointed spaces for $\mathbf{C}=\mathbf{C M}_{\mathbf{n e}}$ and as pointed spaces for $\mathbf{C}=\mathbf{C M}_{\mathbf{0}}, \mathbf{C M}^{2}$.

3. $\mathfrak{h}$ is called strong shape invariant (cf. [23]) if its homology functors $h_{n}$ take strong shape equivalences in $\mathbf{C}$ to isomorphisms in $\mathbf{A b}$.

This is equivalent to the assertion that $\mathfrak{h}$ extends uniquely to a homology theory defined on the appropriate strong shape category. For unreduced homology theories on $\mathbf{C M}^{2}$ it suffices to assume that the $h_{n}$ take strong shape equivalences in $\mathbf{C M} \subset \mathbf{C M}^{2}$ to isomorphisms.

4. $\mathfrak{h}$ is called Holsztynski shape invariant if the $h_{n}$ take (ordinary) shape equivalences in $\mathbf{C}$ to isomorphisms in $\mathbf{A b}$.

This is equivalent to the assertion that $\mathfrak{h}$ extends uniquely to a homology theory defined on the appropriate universal shape category introduced by Holsztynski $[\mathbf{1 0}]$. These categories are not identical with the corresponding (ordinary) shape categories. See [5, Proposition 6.4].

We have defined three kinds of homology theories of strong type and three kinds of Steenrod homology theories living on the three categories C. In [23] we have shown that

- The homology theories of strong type are precisely the strong shape invariant homology theories.

- The three kinds of homology theories of strong type are equivalent by the transformation processes unred and red described in Appendix B. In fact, they occur as associated triples in the sense of Definition B.1.

By looking at these transformation processes we immediately obtain 
Lemma 2.2. Let $\left(\mathfrak{h}^{(n e)}, \mathfrak{h}^{(0)}, \mathfrak{h}\right)$ be an associated triple of homology theories of strong type living on $\mathbf{C M}_{\mathbf{n e}}, \mathbf{C M}_{\mathbf{0}}, \mathbf{C M}^{2}$, respectively. If one component is a Steenrod homology theory, then so are the other two components.

For each Steenrod homology theory on $\mathbf{C}=\mathbf{C M}_{\mathbf{n e}}, \mathbf{C M}_{\mathbf{0}}, \mathbf{C M}^{\mathbf{2}}$ there exists a short exact sequence

(LIM1) $0 \rightarrow \lim ^{1} h_{n+1}(\mathbf{X}) \stackrel{\beta}{\rightarrow} h_{n}(X) \stackrel{\gamma}{\rightarrow} \lim _{\longleftarrow} h_{n}(\mathbf{X}) \rightarrow 0$

for any $X \in \mathbf{C}$ and any inverse sequence $\mathbf{X}$ in $\mathbf{C}$ such that $X=\lim \mathbf{X}$.

This was proved by Milnor [22] for ordinary unreduced Steenrod homology theory and by Kaminker and Schochet [14] for reduced Steenrod homology theories on $\mathbf{C M}_{\text {ne }}$. Milnor's proof does not refer to a specific construction of ordinary Steenrod homology but is based on the axioms except the dimension axiom. The proof is therefore valid also for generalized unreduced Steenrod homology theories and can easily be transferred to the case of reduced Steenrod homology theories on $\mathbf{C M}_{\mathbf{n e}}$ and $\mathbf{C M}_{\mathbf{0}}$ as it was done in [14]. However, two important issues have not been addressed so far.

1. $\beta$ and $\gamma$ are constructed by a lot of diagram chasing which does not make transparent what these homomorphisms "really" look like. In particular, it remains open whether one may take $\gamma=\mathbf{p}_{\#}$ (which is the canonical candidate).

2. The categorical framework of the $\lim ^{1}$-sequence needs to be clarified. On which category of inverse sequences do the three functors in the $\lim ^{1}$-sequence live and are $\beta$ and $\gamma$ natural? On the category $\mathbf{C}^{\mathbb{N}}$ of level maps between inverse sequences everything works smoothly - the naturalness of $\beta$ and $\gamma$ follows immediately from their construction. On the pro-category of towers tow-C (cf. Appendix A) as the "optimal habitat" of the above three functors the naturalness of $\beta$ and $\gamma$ is not obvious and has not been established in the literature (the proof of [14, Proposition 2.11] contains a gap - in the crucial case of cofinal subsequences it does not incorporate how the identifications of the $\lim ^{1}$-terms and lim-terms work exactly and fails to show that $\beta$ and $\gamma$ respect these identifications).

In this paper we clarify these issues and supply a refined version of the $\lim ^{1}$-sequence. As a consequence we shall see that Steenrod homology theories are nothing else than partially continuous homology theories. See Definition 3.1 and Theorem 3.7 below.

\section{Partially continuous homology theories}

The axiom of partial continuity was introduced in [12] (see also [11]). We need it in a slightly modified form. Let $\mathbf{A N R}(\mathbf{C M}), \mathbf{A N R}\left(\mathbf{C M}_{\mathbf{n e}}\right), \mathbf{A N R}\left(\mathbf{C M}_{\mathbf{0}}\right)$ and $\operatorname{ANR}\left(\mathbf{C M}^{\mathbf{2}}\right)$ denote the categories of compact ANRs, compact nonempty ANRs, compact pointed ANRs and pairs of compact ANRs, respectively.

In the sequel let $\mathbf{C}$ denote one of the categories $\mathbf{C M}_{\text {ne }}, \mathbf{C M}_{\mathbf{0}}, \mathbf{C M}^{\mathbf{2}}$ and let tow- $\mathbf{C}$ denote the category of towers over $\mathbf{C}$ (cf. [8]) which is a subcategory of the category of inverse systems pro- $\mathbf{C}$ over $\mathbf{C}$. The inverse limit is a functor

$$
\underset{\longleftarrow}{\lim }: \text { tow- } \mathbf{C} \rightarrow \mathbf{C}
$$

coming along with a natural transformation $\mathbf{p}: X=\lim \mathbf{X} \rightarrow \mathbf{X}$ in pro-C. For any $\mathbf{X}=\left(X_{i}, p_{i}: X_{i+1} \rightarrow X_{i}\right)_{i \geqslant 1}$ in tow-C we write $\mathbf{p}=\left(p_{i}^{\infty}: X \rightarrow X_{i}\right)$. $\mathbf{p}$ induces a 
morphism $\mathbf{p}_{*}: h_{n}(X) \rightarrow h_{n}(\mathbf{X})=\left(h_{n}\left(X_{i}\right),\left(p_{i}\right)_{*}\right)$ in pro-Ab and thus a homomorphism $\mathbf{p}_{\#}=\lim \mathbf{p}_{*}: h_{n}(X) \rightarrow \lim _{\longleftarrow} h_{n}(\mathbf{X})$ which is clearly a natural transformation $h_{n} \circ \lim _{\longleftarrow} \rightarrow \lim _{\longleftarrow} h_{n}$ of functors on tow-C.

Definition 3.1. Let $\mathbf{C}^{\prime} \subset \mathbf{C}$ be a full subcategory. A homology theory $\mathfrak{h}$ on $\mathbf{C}$ with homology functors $h_{n}$ is called $\mathbf{C}^{\prime}$-partially continuous if there exist natural transfor-

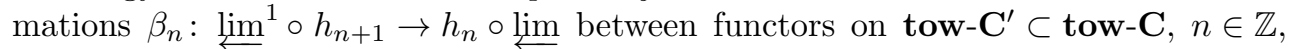
such that the following sequence is exact for all $\mathbf{X} \in$ tow- $\mathbf{C}^{\prime}$ :

$$
0 \rightarrow \lim ^{1} h_{n+1}(\mathbf{X}) \stackrel{\beta_{n}}{\rightarrow} h_{n}(X) \stackrel{\mathbf{p}_{\#}}{\rightarrow} \lim _{\longleftarrow} h_{n}(\mathbf{X}) \rightarrow 0 .
$$

A C-partially continuous homology theory is called maximally partially continuous. An $\mathbf{A N R}(\mathbf{C})$-partially continuous homology theory is called ANR-partially continuous.

Proposition 3.2. $\quad$ 1. Each ANR-partially continuous homology theory is Holsztynski shape invariant and therefore of strong type. In case $\mathbf{C}=\mathbf{C M}^{2}$ it suffices to assume $\mathbf{A N R}(\mathbf{C M})$ - or $\mathbf{A N R}\left(\mathbf{C M}_{\mathbf{0}}\right)$-partial continuity.

2. Each maximally partially continuous homology theory is a Steenrod homology theory.

Proof. (1) For any map $f: X \rightarrow Y$ in $\mathbf{C}$ there exists a morphism $\mathbf{f}: \mathbf{X} \rightarrow \mathbf{Y}$ in tow-ANR(C) such that $X=\lim \mathbf{X}, Y=\lim \mathbf{Y}$ and $f=\lim \mathbf{f}$. The map $f$ is a shape equivalence in $\mathbf{C}$ if and only if $\overleftarrow{\mathbf{f}}$ induces $\overleftarrow{\mathrm{nn}}$ isomorphism in the pro-homotopy category tow-Ho $(\mathbf{C})$. The functors $h_{k}$ : tow- $\mathbf{C} \rightarrow \mathbf{A b}$ (and a fortiori also the functors $\varliminf^{1} \circ h_{k}$ and $\lim \circ h_{k}$ ) factorize through tow-Ho(C). Thus if $f$ is a shape equivalence, then the above $\mathbf{f}$ induces isomorphisms $\mathbf{f}_{*}: \lim ^{1} h_{n+1}(\mathbf{X}) \rightarrow \lim ^{1} h_{n+1}(\mathbf{Y})$ and $\mathbf{f}_{*}: \lim h_{n}(\mathbf{X}) \rightarrow \lim h_{n}(\mathbf{Y})$. The five lemma shows therefore that ANR-partially continuous homology theories are Holsztynski shape invariant and a fortiori strong shape invariant (cf. proof of [5, Proposition 6.1]).

Consider $\mathbf{C}=\mathbf{C M}^{2}$. The above argument shows that (a) $\mathbf{A N R}(\mathbf{C M})$-partial continuity resp. (b) $\mathbf{A N R}\left(\mathbf{C M}_{\mathbf{0}}\right)$-partial continuity implies that the following types of maps induce isomorphisms in homology: (a) shape equivalences $f: X \rightarrow Y$ between single spaces resp. (b) pointed shape equivalences $f:\left(X, x_{0}\right) \rightarrow\left(Y, y_{0}\right)$. In case (a) the five lemma applied to the long exact sequences of pairs covers the general case of shape equivalences $f:(X, A) \rightarrow(Y, B)$. Moreover, (b) implies (a): Any shape equivalence $f: X \rightarrow Y$ yields a pointed shape equivalence $f^{+}:\left(X^{+}, *\right) \rightarrow\left(Y^{+}, *\right)$ which induces isomorphisms in homology. Excision shows that $f$ itself induces an isomorphism in homology.

(2) The cluster axiom for maximally partially continuous homology theories follows from the fact that a cluster is the inverse limit of a tower of finite wedges (which is movable and thus has a vanishing $\lim ^{1}$-term; see the proof of [8, Corollary 8.5.3]).

Remark 3.3. The only property of the functors $\mathfrak{F}_{n}=\lim ^{1} \circ h_{n+1}$ : tow-C $\rightarrow \mathbf{A b}$ used in the proof of part (1) of the above proposition is that they factorize through the pro-homotopy category tow-Ho(C). The only property used in the proof of (2) is that they vanish on the class of movable towers. An essential ingredient in the proof of (2) is that the exact $\lim ^{1}$-sequence contains $\mathbf{p}_{\#}$. Working with an abstract $\gamma$ would not show that the cluster homomorphism $\Pi$ is an isomorphism. 
Remark 3.4. An alternative proof of part (2) can be given by showing as in the proof [20, Theorem 1.2] that the strong excision axiom is satisfied instead of using part (1).

Lemma 3.5. For a homology theory $\mathfrak{h}$ on $\mathbf{C M}^{2}$ the following are equivalent:

1. $\mathfrak{h}$ is maximally partially continuous (resp. ANR-partially continuous).

2. $\mathfrak{h}$ is $\mathbf{C M}$-partially continuous (resp. $\mathbf{A N R}(\mathbf{C M})$-partially continuous).

3. $\mathfrak{h}$ is $\mathbf{C M}_{\mathbf{0}}$-partially continuous (resp. $\mathbf{A N R}\left(\mathbf{C M}_{\mathbf{0}}\right)$-partially continuous).

Proof. (1) $\Rightarrow(2)$ : Trivial.

$(2) \Rightarrow(3)$ (cf. proof of $\left[\mathbf{2 2}\right.$, Theorem 4]): For objects of $\mathbf{C M}_{\mathbf{0}}$ there is a natural isomorphism $h_{n}(Z) \approx h_{n}\left(Z, z_{0}\right) \oplus h_{n}\left(z_{0}\right)$. Let $\mathbf{X}=\left(\left(X_{i}, x_{i 0}\right), p_{i}\right)$ be a tower in $\mathbf{C M}_{\mathbf{0}} \subset \mathbf{C M}^{\mathbf{2}}$ and let $\mathbf{X}^{\prime}=\left(X_{i}, p_{i}\right)$ denote the tower in tow-CM obtained by forgetting basepoints. If $\mathbf{p}:\left(X, x_{0}\right)=\lim \mathbf{X} \rightarrow \mathbf{X}$ is the limit morphism in pro- $\mathbf{C M}_{\mathbf{0}}$, we denote by $\mathbf{p}^{\prime}: X=\lim _{\mathbf{X}^{\prime}} \rightarrow \mathbf{X}^{\prime}$ the limit morphism in pro-CM which is identical with $\mathbf{p}$ if basepoints are forgotten. There is a commutative diagram with exact top row

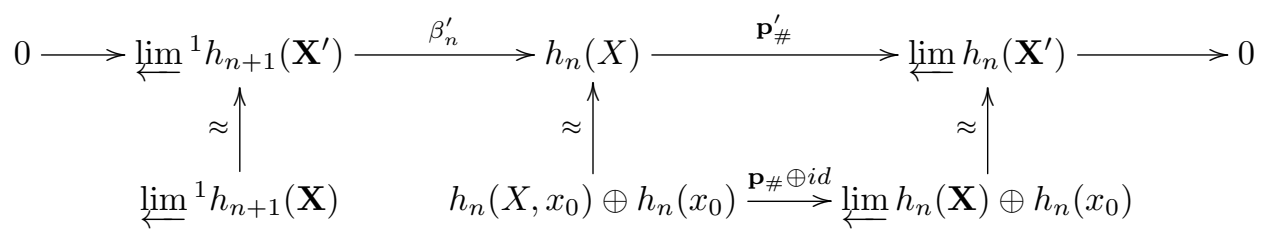

Because the kernel of $\mathbf{p}_{\#}^{\prime}$ can be identified with $\operatorname{ker}\left(\mathbf{p}_{\#}\right) \oplus 0$, we have $\operatorname{im}\left(\beta_{n}^{\prime}\right)=$ $\operatorname{ker}\left(\mathbf{p}_{\#}\right) \oplus 0$ so that $\beta_{n}^{\prime}$ induces a natural homomorphism $\beta_{n}: \lim ^{1} h_{n+1} \mathbf{X} \rightarrow h_{n}\left(X, x_{0}\right)$ making the appropriate sequence exact.

$(3) \Rightarrow(1)$ : $\mathfrak{h}$ satisfies the strong excision axiom by Proposition 3.2. Let $\mathbf{X}=$ $\left(\left(X_{i}, A_{i}\right), p_{i}\right)$ be a tower in $\mathbf{C M}^{2}$. Then $\mathbf{X}^{\prime}=\left(\left(X_{i} / A_{i}, *\right), p_{i}^{\prime}\right)$ is a tower in $\mathbf{C M}_{\mathbf{0}}$, and if all $\left(X_{i}, A_{i}\right)$ are ANR-pairs, then all $\left(X_{i} / A_{i}, *\right)$ are pointed ANRs. The natural level quotient map q: $\mathbf{X} \rightarrow \mathbf{X}^{\prime}$ induces isomorphisms $\lim ^{1} \mathbf{q}_{*}: \lim ^{1} h_{n+1}(\mathbf{X}) \rightarrow$ $\varliminf^{1} h_{n+1}\left(\mathbf{X}^{\prime}\right)$ and $\varliminf_{\lim } \mathbf{q}_{*}: \lim _{n+1}(\mathbf{X}) \rightarrow \varliminf_{\lim } h_{n+1}\left(\mathbf{X}^{\prime}\right)$. The $\operatorname{limit}_{n a p} \varlimsup_{\mathbf{l i m}} \mathbf{q}:(X, A)=$ $\varlimsup \mathbf{l i m} \mathbf{X} \rightarrow \varliminf_{\lim } \mathbf{X}^{\prime}=(\overleftarrow{X} / A, *)$ is nothing else than the quotient map and thus also induces isomorphisms in homology. This proves (1).

Lemma 3.6. Let $\left(\mathfrak{h}^{(n e)}, \mathfrak{h}^{(0)}, \mathfrak{h}\right)$ be an associated triple of homology theories of strong type living on $\mathbf{C M}_{\mathbf{n e}}, \mathbf{C M}_{\mathbf{0}}, \mathbf{C M}^{\mathbf{2}}$, respectively. If one component is a maximally partially continuous homology theory (resp. an ANR-partially continuous homology theory), then so are the other two components.

Proof. Consulting Appendix B, it is clear that maximal partial continuity of $\mathfrak{h}^{(0)}$ is equivalent to $\mathbf{C M}_{\mathbf{0}}$-partial continuity of $\mathfrak{h}$ and hence by Lemma 3.5 to maximal partial continuity of $\mathfrak{h}$. Similarly, maximal partial continuity of $\mathfrak{h}^{(n e)}$ implies CMpartial continuity of $\mathfrak{h}$ and thus maximal partial continuity of $\mathfrak{h}$. It remains to show that if $\mathfrak{h}$ is maximally partially continuous, then $\operatorname{red}(\mathfrak{h}) \approx \mathfrak{h}^{(n e)}$ is maximally partially continuous. There is a natural isomorphism $h_{n}(X) \approx h_{n}^{(n e)}(X) \oplus h_{n}(*)$. The argument goes now as in the proof of Lemma 3.5.

Here is the main result of this paper. 
Theorem 3.7. A homology theory $\mathfrak{h}$ is maximally partially continuous if and only if it is a Steenrod homology theory.

Proof. It remains to show that Steenrod homology theories are maximally partially continuous. We shall do this by proving that Milnor's original $\lim ^{1}$-sequence is indeed a sequence as required in Definition 3.1. Since each Steenrod homology theory occurs as a component of an associated triple, Lemmas 2.2, 3.5 and 3.6 imply that it suffices to show that a Steenrod homology theory $\mathfrak{h}=\left(h_{n}, \partial_{n}\right)$ on $\mathbf{C M}^{\mathbf{2}}$ is CM-partially continuous.

We begin with some notation. Each inverse sequence $\mathbf{X}=\left(X_{i}, p_{i}\right)_{i \geqslant 1}$ in $\mathbf{C M}$ can be augmented to a sequence $\left(X_{i}, p_{i}\right)_{i \geqslant 0}$ with $X_{0}=*$ and $p_{0}: X_{1} \rightarrow X_{0}$ the unique map. Morphisms $\mathbf{f}=\left(\varphi, f_{i}\right): \mathbf{X} \rightarrow \mathbf{Y}$ in seq-CM (cf. Appendix A) can be extended to the augmented sequences by letting $\varphi(0)=0$ and $f_{0}: X_{0} \rightarrow Y_{0}$ be the unique map.

Let $X=\lim \mathbf{X}$. The contractible telescope $T=T \mathbf{X}$ as constructed in $[\mathbf{1 6}]$ is a

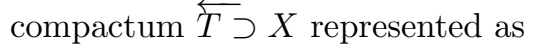

$$
T=M_{0} \cup M_{1} \cup M_{2} \cup \cdots \cup X,
$$

such that

1. $M_{i}$ is a copy of the mapping cylinder of $p_{i}$ having $X_{i}$ as the base and $X_{i+1}$ as the top,

2. $M_{i} \cap M_{i+1}=X_{i+1}$,

3. $M_{i} \cap M_{j}=\emptyset$ for $j>i+1$,

4. $M_{i} \cap X=\emptyset$.

There are strong deformation retractions

$$
r_{i}: T \rightarrow T_{i}= \begin{cases}X_{0} & \text { for } i=0, \\ M_{0} \cup \cdots \cup M_{i-1} & \text { for } i>0\end{cases}
$$

uniquely characterized by

$$
\begin{gathered}
\left.r_{i}\right|_{M_{j}}=r_{i}^{j}: M_{j} \stackrel{\text { retr }}{\rightarrow} X_{j} \stackrel{p_{i}^{j}}{\rightarrow} X_{i} \subset T_{i} \text { for } j \geqslant i, \\
\left.r_{i}\right|_{X}=p_{i}^{\infty}: X \rightarrow X_{i} \subset T_{i} .
\end{gathered}
$$

Let us define a compact subspace $|\mathbf{X}| \subset T \mathbf{X}$ by

$$
|\mathbf{X}|=X_{0} \cup X_{1} \cup X_{2} \cup \cdots \cup X \text {. }
$$

This extends to a functor

$$
|-|: \text { seq-CM } \rightarrow \mathbf{C M},
$$

such that for a morphism $\mathbf{f}=\left(\varphi, f_{i}\right): \mathbf{X} \rightarrow \mathbf{Y}$

$$
\left.|\mathbf{f}|\right|_{X}=\lim _{\longleftarrow} \mathbf{f} \text { and }\left.|\mathbf{f}|\right|_{X_{j}}=f_{i} \circ p_{\varphi(i)}^{j} \text { for } \varphi(i) \leqslant j<\varphi(i+1) .
$$

Also $T$ is a functor on seq-CM, but this is irrelevant for our purposes.

Let us define compact subspaces $T^{1}, T^{2} \subset T$ by

$$
\begin{aligned}
& T^{1}=X_{0} \cup M_{1} \cup M_{3} \cup \cdots \cup X, \\
& T^{2}=M_{0} \cup M_{2} \cup M_{4} \cup \cdots \cup X .
\end{aligned}
$$


We have $T^{1} \cup T^{2}=T$ and $T^{1} \cap T^{2}=|\mathbf{X}|$. For $i \geqslant 1$ set

$$
R_{i}=X_{i} \cup X_{i+1} \subset M_{i} \text { with inclusions } l_{k}^{i}: X_{k} \rightarrow R_{i}, k=i, i+1 .
$$

For any compact $Z \subset T-X_{0}=T-*$, let $Z^{+}=Z \cup *$. Concerning diagrams of homology groups we adopt the following conventions:

- Unnamed arrows are induced by inclusions.

- Isomorphisms are characterized by the symbol $\approx$.

- Unnamed isomorphisms are inclusion-induced excision isomorphisms.

- Isomorphisms named by $p_{*}$ are strong excision isomorphisms induced by quotient maps $p$.

- Isomorphisms named by $h_{*}^{(Z, C)}$ are induced by a canonical homeomorphism $h^{(Z, C)}$ from $(Z / C, *)$ to an appropriate cluster.

By Milnor's construction in [22], the exact sequence (LIM1) is obtained by using the Mayer-Vietoris sequence of the excisive triad $\left(T ; T^{1}, T^{2}\right)$ with subspace $X^{+} \subset|\mathbf{X}|=$ $T^{1} \cap T^{2}$ and the exact sequence of the triple $\left(T, X^{+}, *\right)$. We recall the construction of $\gamma$ :

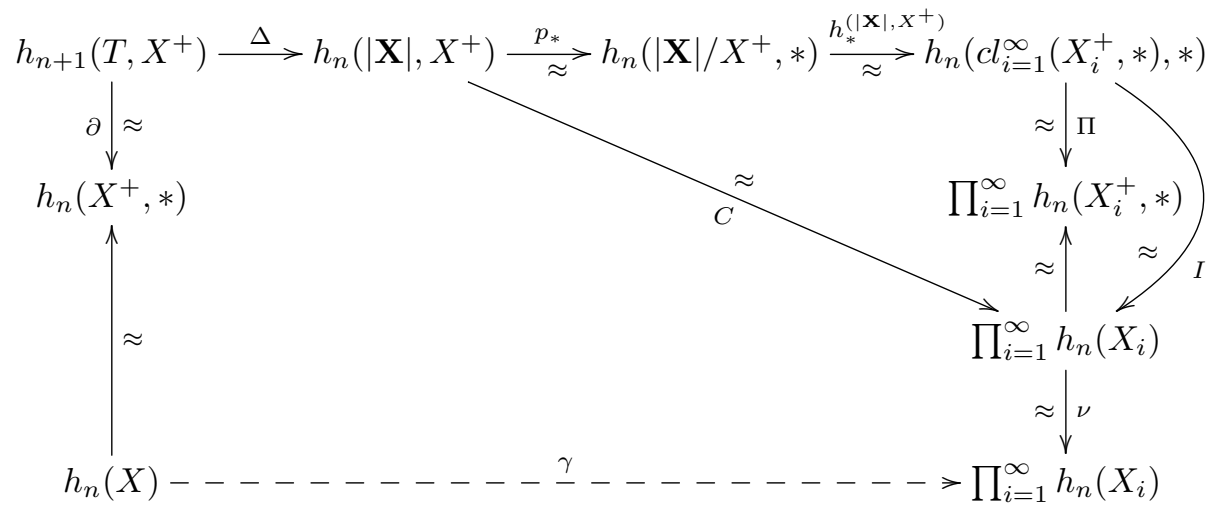

$\Delta$ is part of the Mayer-Vietoris sequence and the automorphism $\nu$ is given by $\nu\left(\left(x_{i}\right)\right)=$ $\left((-1)^{i} x_{i}\right)$. The isomorphisms $C$ and $I$ are defined as the appropriate compositions. It is shown in $[\mathbf{2 2}]$ that the map $\gamma$ occurring as the unique filler is an epimorphism onto $\lim _{h}(\mathbf{X}) \subset \prod_{i=1}^{\infty} h_{n}\left(X_{i}\right)$. Let us also recall that $\Delta$ is the following composition

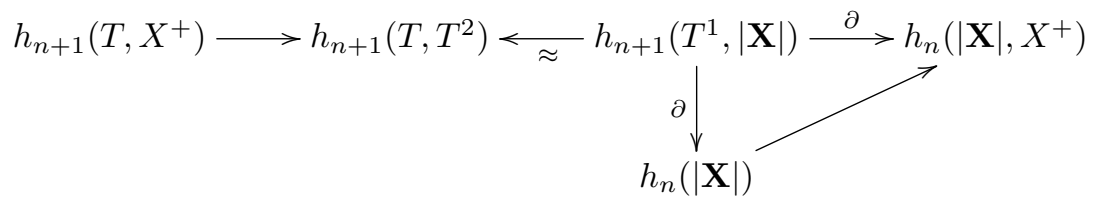

Here, the horizontal $\partial$ comes from exact sequence of the triple $\left(T^{1},|\mathbf{X}|, X^{+}\right)$and the vertical $\partial$ from exact sequence of the pair $\left(T^{1},|\mathbf{X}|\right)$.

Our objective is to prove that $\gamma=\mathbf{p}_{\#}$. To do this it suffices to show that for each odd $j$ 


$$
\left(\psi_{j} \circ \nu \circ \gamma\right)(x)=\left(-\left(p_{j}^{\infty}\right)_{*}(x),\left(p_{j+1}^{\infty}\right)_{*}(x)\right) \in h_{n}\left(X_{j}\right) \times h_{n}\left(X_{j+1}\right),
$$

with projection $\psi_{j}: \prod_{i=1}^{\infty} h_{n}\left(X_{i}\right) \rightarrow h_{n}\left(X_{j}\right) \times h_{n}\left(X_{j+1}\right)$.

For a given odd $j$, let $\rho_{j}:\left(T^{1},|\mathbf{X}|\right) \rightarrow\left(M_{j}^{+}, R_{j}^{+}\right)$be the retraction sending $T^{1}-M_{j}$ to $*$ and $q_{j}:\left(T, T^{2}\right) \rightarrow\left(M_{j} / R_{j}, *\right)$ be the map which is the identity on int $M_{j}$ and collapses $T-i n t M_{j}$ to a point. Moreover, let $\alpha_{j}: h_{n}\left(X_{j} \cup X_{j+1}\right) \rightarrow h_{n}\left(X_{j}\right) \times h_{n}\left(X_{j+1}\right)$ be the isomorphism given by $\left(\alpha_{j}\right)^{-1}(g, h)=\left(l_{j}^{j}\right)_{*}(g)+\left(l_{j+1}^{j}\right)_{*}(h)$. We obtain the following two commutative diagrams:

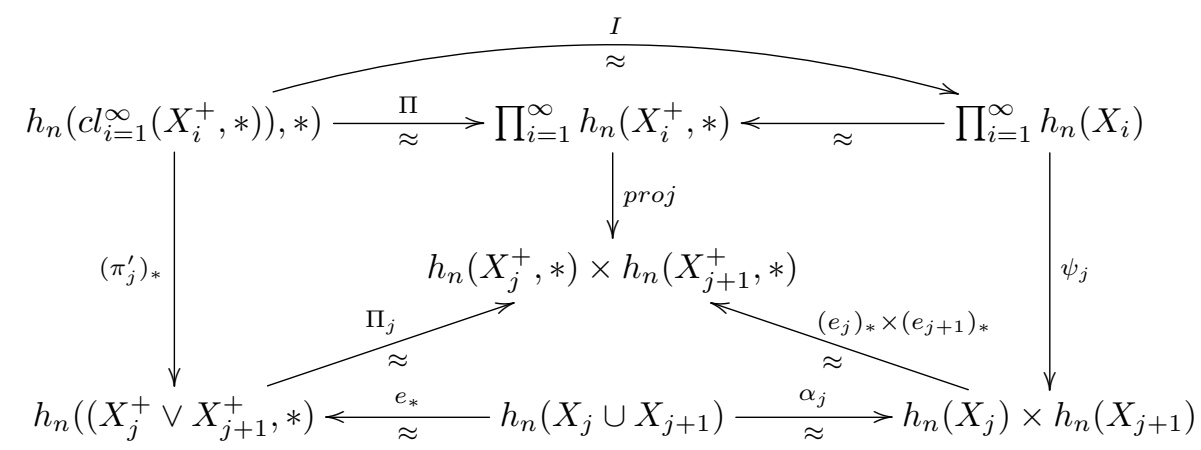

Here, $e, e_{j}, e_{j+1}$ denote excisions. We have $\Pi_{j} e_{*}(x)=\left(\left(\hat{\pi}_{j}\right)_{*} e_{*}(x),\left(\hat{\pi}_{j+1}\right)_{*} e_{*}(x)\right)$ so that

$$
\begin{aligned}
\Pi_{j} e_{*} \alpha_{j}^{-1}(g, h)= & \left(\left(\hat{\pi}_{j}\right)_{*} e_{*}\left(l_{j}^{j}\right)_{*}(g)+\left(\hat{\pi}_{j}\right)_{*} e_{*}\left(l_{j+1}^{j}\right)_{*}(h),\left(\hat{\pi}_{j+1}\right)_{*} e_{*}\left(l_{j}^{j}\right)_{*}(g)\right. \\
& +\left(\left(\hat{\pi}_{j+1}\right)_{*} e_{*}\left(l_{j+1}^{j}\right)_{*}(h)\right) \\
= & \left(\left(e_{j}\right)_{*}(g),\left(e_{j+1}\right)_{*}(g)\right)
\end{aligned}
$$

because $\hat{\pi}_{k} e l_{k^{\prime}}^{j}=e_{k}$ for $k=k^{\prime}$ and $\hat{\pi}_{k} e l_{k^{\prime}}^{j}=c$ for $k \neq k^{\prime}$, where $c$ maps $X_{k^{\prime}}$ to $* \in X_{k}^{+}$ and thus induces the zero map in homology.

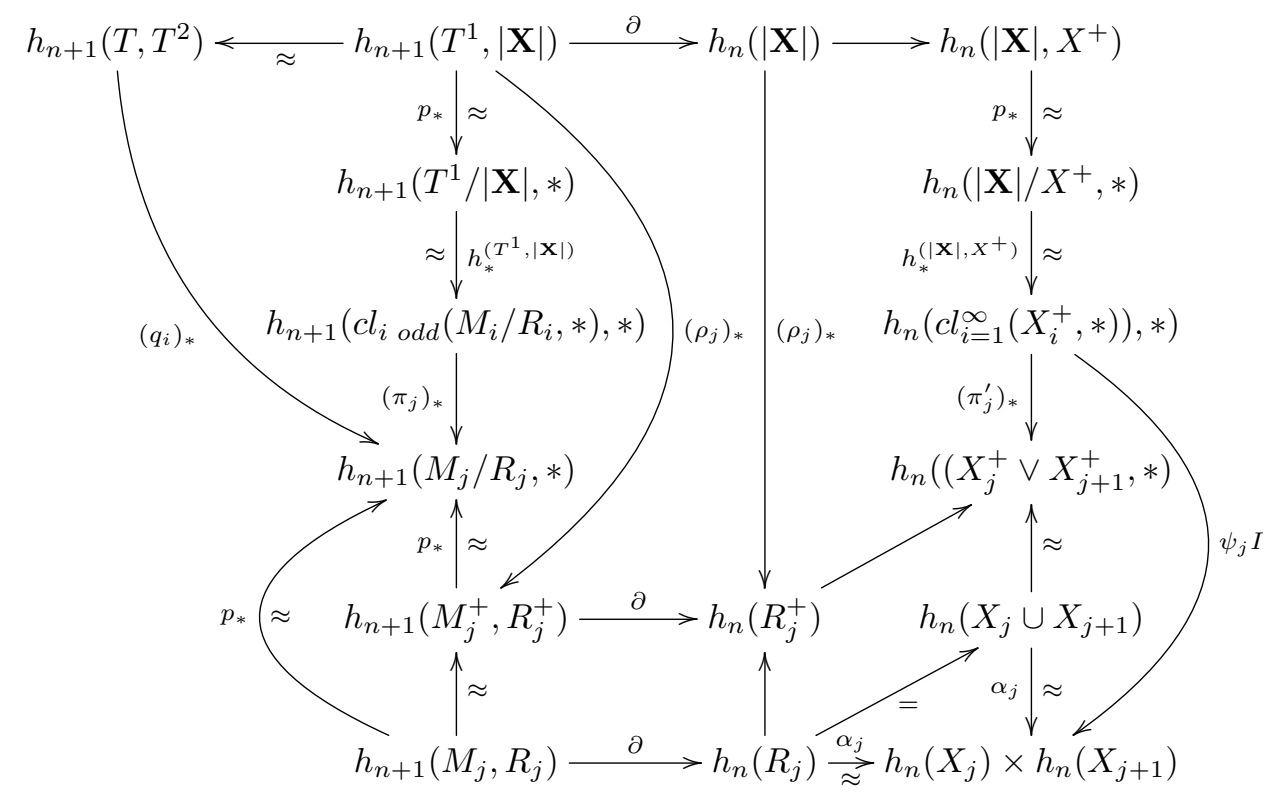


In particular, we can conclude that the image of $\psi_{j} \circ \nu \circ \gamma$ is contained in the image of $\alpha_{j} \circ \partial: h_{n+1}\left(M_{j}, R_{j}\right) \rightarrow h_{n}\left(X_{j}\right) \times h_{n}\left(X_{j+1}\right)$. We claim that $i m\left(\alpha_{j} \circ \partial\right)$ $=\alpha_{j}(i m(\partial))=\left\{(g, h) \in h_{n}\left(X_{j}\right) \times h_{n}\left(X_{j+1}\right) \mid g=-\left(p_{j}\right)_{*}(h)\right\}$. To see this, let $r: M_{j}$ $\rightarrow X_{j}$ be a strong deformation retraction and $l: R_{j} \rightarrow M_{j}$ the inclusion. We have $\alpha_{j}^{-1}(g, h) \in \operatorname{im}(\partial)$ iff $\left(l_{j}^{j}\right)_{*}(g)+\left(l_{j+1}^{j}\right)_{*}(h) \in \operatorname{im}(\partial)=\operatorname{ker}\left(l_{*}\right)=k e r\left((r l)_{*}\right)$ iff $g+\left(p_{j}\right)_{*}(h)=(r l)_{*}\left(\left(l_{j}^{j}\right)_{*}(g)\right)+(r l)_{*}\left(\left(l_{j+1}^{j}\right)_{*}(h)=0\right.$. It therefore suffices to prove

$$
\left(\operatorname{proj} \circ \psi_{j} \circ \nu \circ \gamma\right)(x)=\left(p_{j+1}^{\infty}\right)_{*}(x) \in h_{n}\left(X_{j+1}\right),
$$

with projection proj: $h_{n}\left(X_{j}\right) \times h_{n}\left(X_{j+1}\right) \rightarrow h_{n}\left(X_{j+1}\right)$. We have the following commutative diagram:

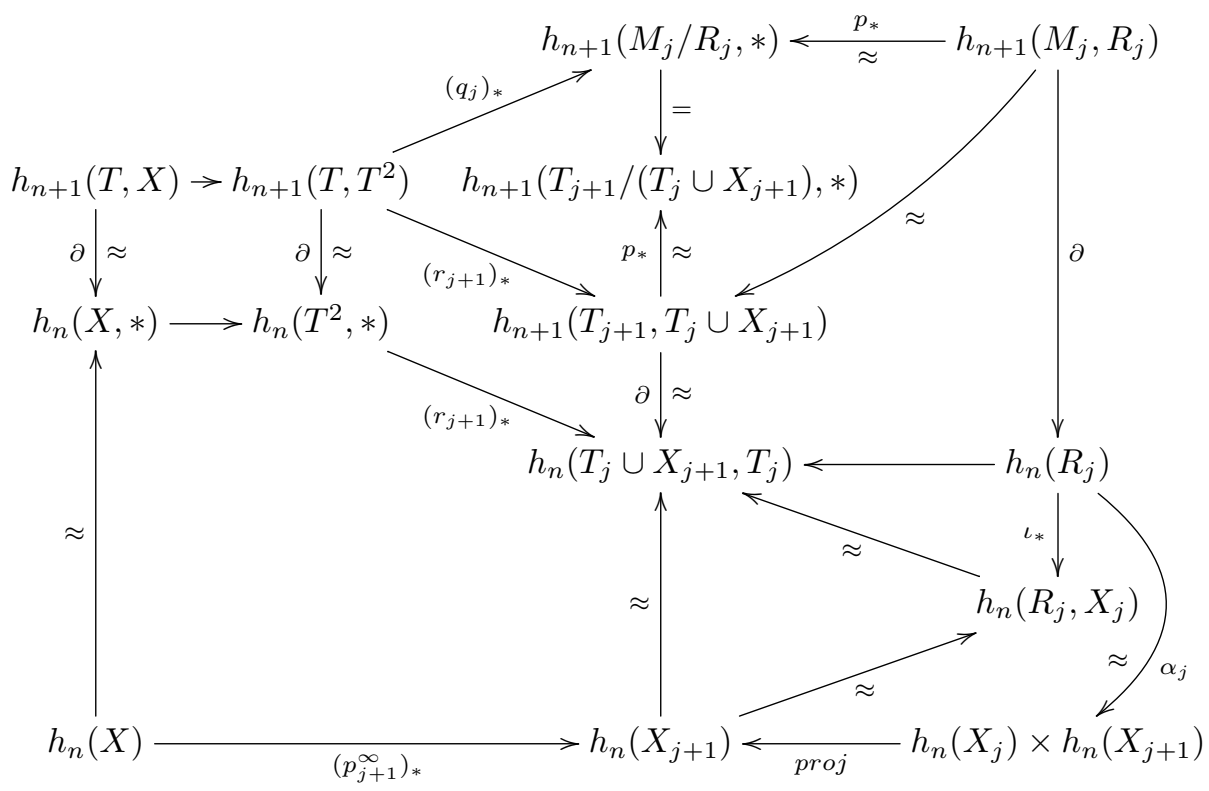

In the lower right square note $\iota_{*}\left(\left(\alpha_{j}\right)^{-1}(g, h)\right)=\left(\iota l_{j}^{j}\right)_{*}(g)+\left(\iota l_{j+1}^{j}\right)_{*}(h)=\left(\iota l_{j+1}^{j}\right)_{*}(h)$ $=\left(\iota l_{j+1}^{j}\right)_{*}(\operatorname{proj}(g, h))$ since $\left(\iota l_{j}^{j}\right)_{*}: h_{n}\left(X_{j}\right) \rightarrow h_{n}\left(R_{j}, X_{j}\right)$ is zero.

Putting all pieces together we obtain the desired equation

$$
\left.\left(\operatorname{proj} \circ \psi_{j} \circ \nu \circ \gamma\right)(x)\right)=\left(p_{j+1}^{\infty}\right)_{*}(x) .
$$

It remains to show that the map $\beta: \lim ^{1} h_{n+1}(\mathbf{X}) \rightarrow h_{n}(X)$ in Milnor's exact sequence (LIM1) is natural. We recall its construction. For this purpose let

$$
\begin{aligned}
& |\mathbf{X}|^{1}=* \cup X_{1} \cup X_{3} \cup \cdots \cup X, \\
& |\mathbf{X}|^{2}=* \cup X_{2} \cup X_{4} \cup \cdots \cup X .
\end{aligned}
$$

The inclusions $|\mathbf{X}|^{k} \rightarrow T^{k}$ embed $|\mathbf{X}|^{k}$ as strong deformation retracts into $T^{k}$. In the following commutative diagram the top row belongs to the Mayer-Vietorissequence of the excisive triad $\left(T ; T^{1}, T^{2}\right)$ with subspace $X^{+} \subset|\mathbf{X}|=T^{1} \cap T^{2}$. Explicitly we have $u(x)=\left(-\operatorname{incl}_{*}^{1}(x), \operatorname{incl}_{*}^{2}(x)\right)$ and the maps $s$ are given as $s(y, z)=$ $i n c l_{*}^{1}(y)+i n c l_{*}^{2}(z)$ with the appropriate inclusions $i n c l^{k}$. 


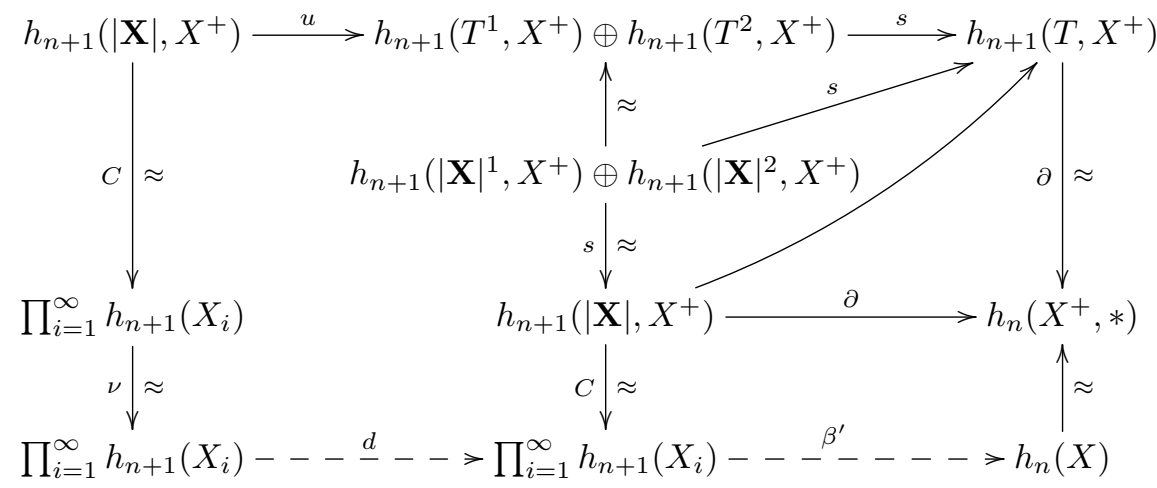

The maps $d$ and $\beta^{\prime}$ are defined as the unique fillers. It is easily verified that (cf. [22]) $d\left(\left(\xi_{i}\right)\right)=\left(\xi_{i}-\left(p_{i}\right)_{*}\left(\xi_{i+1}\right)\right)$ so that the cokernel of $d$ is nothing else than $\lim ^{1} h_{n+1}(\mathbf{X})$. Hence $\beta^{\prime}$ induces a monomorphism $\beta: \lim ^{1} h_{n+1}(\mathbf{X}) \rightarrow h_{n}(X)$.

Define two functors $\Theta, \Omega$ : seq- $\mathbf{C M} \rightarrow \mathbf{C M}^{\mathbf{2}}$ by $\Theta(\mathbf{X})=h_{n+1}\left(|\mathbf{X}|, X^{+}\right), \Theta(\mathbf{f})=$ $|\mathbf{f}|_{*}$ and $\Omega(\mathbf{X})=\prod_{i=1}^{\infty} h_{n+1}\left(X_{i}\right),(\Omega(\mathbf{f})(g))_{i}=\sum_{j=\varphi(i)}^{\varphi(i+1)-1}\left(f_{i} p_{\varphi(i)}^{j}\right)_{*}\left(g_{j}\right)$, where $\mathbf{f}=$ $\left(\varphi, f_{i}\right): \mathbf{X} \rightarrow \mathbf{Y}$.

The isomorphism $C: \Theta(\mathbf{X}) \rightarrow \Omega(\mathbf{X})$ is easily seen to be natural on seq-CM. Since $\partial: \Theta(\mathbf{X}) \rightarrow h_{n}\left(X^{+}, *\right)$ is natural on seq-CM, the same is true for $\beta^{\prime}: \Omega(\mathbf{X}) \rightarrow h_{n}(X)$.

Let $\mathbf{f}: \mathbf{X} \rightarrow \mathbf{Y}$ be a morphism in seq-CM and let $\mathbf{f}_{*}: h_{n+1}(\mathbf{X}) \rightarrow h_{n+1}(\mathbf{Y})$ be the induced morphism in seq-Ab. We have $\Omega(\mathbf{f})=L^{1}\left(\mathbf{f}_{*}\right)$, where $L^{1}$ is defined in Appendix A. We therefore have a commutative diagram

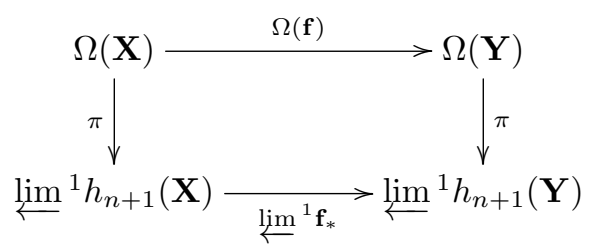

Since $\pi$ is an epimorphism, also $\beta$ is natural on seq-CM. The naturalness on tow-CM follows from the fact that equivalent morphisms in seq-CM induce the same morphism between the domains and ranges of $\beta$, respectively.

The strong excision axiom and the cluster axiom produce an isomorphism $C^{\prime}: h_{n+1}(|\mathbf{X}|, X) \rightarrow \prod_{i=0}^{\infty} h_{n+1}\left(X_{i}\right)$ which extends the above $C$ in the sense that

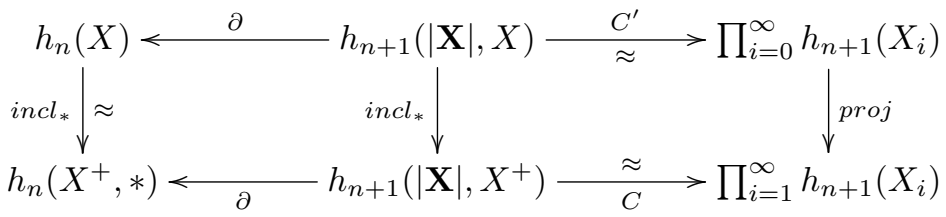

is a commutative diagram which is natural on seq-CM. Let $\pi^{\prime}: \prod_{i=0}^{\infty} h_{n+1}\left(X_{i}\right) \stackrel{\text { proj }}{\rightarrow}$ $\prod_{i=1}^{\infty} h_{n+1}\left(X_{i}\right) \stackrel{\pi}{\rightarrow} \lim ^{1} h_{n+1}(\mathbf{X})$.

Corollary 3.8. For any Steenrod homology theory $\mathfrak{h}$ on $\mathbf{C M}^{2}$ the natural transformation $\beta: \lim ^{1} h_{n+1}(\mathbf{X}) \rightarrow h_{n}(X)$ can be chosen as the unique filler in the following 
diagram (which is natural on $\mathbf{s e q - C M}$ ):

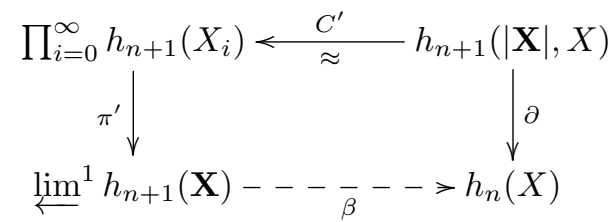

Hence there is a commutative diagram which is natural on seq-CM:

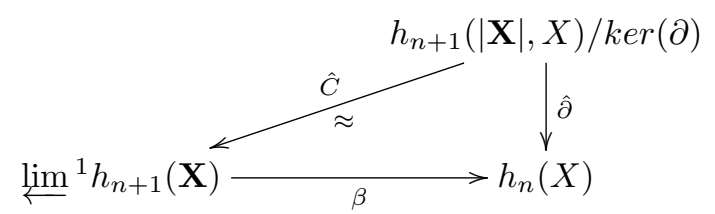

Thus $\beta$ is essentially the map induced by the boundary operator $\partial: h_{n+1}(|\mathbf{X}|, X) \rightarrow$ $h_{n}(X)$ on its coimage $\operatorname{coim}(\partial)=h_{n+1}(|\mathbf{X}|, X) / \operatorname{ker}(\partial)$.

Proof. That $\beta$ can be chosen as a filler follows from the above proof and the definition of $C^{\prime}$. The uniqueness of $\beta$ is trivial since $\pi^{\prime}$ is an epimorphism. Since $\beta$ is a monomorphism, we have $\operatorname{ker}\left(\pi^{\prime} C^{\prime}\right)=\operatorname{ker}(\partial)$. This produces the second diagram.

There is a commutative diagram with exact rows

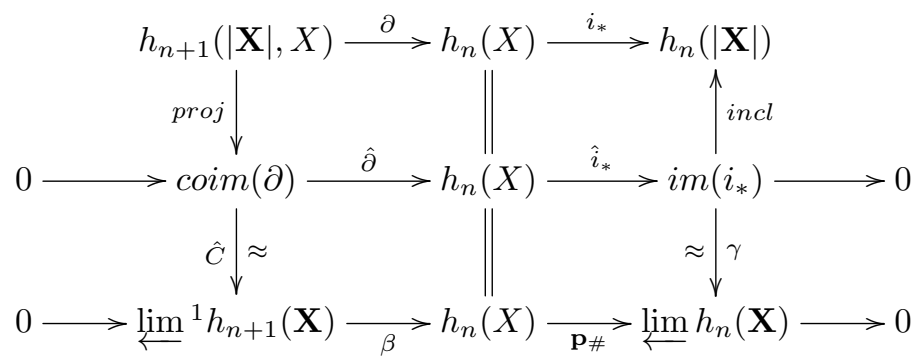

where $\gamma$ is the composition of the canonical isomorphisms $i m\left(i_{*}\right) \approx h_{n}(X) / \operatorname{ker}\left(i_{*}\right)=$ $h_{n}(X) / \operatorname{ker}\left(\mathbf{p}_{\#}\right) \approx \lim h_{n}(\mathbf{X})$. This yields a nice alternative interpretation of the $\lim ^{1}$ sequence. It also shows that $i_{*}: h_{n}(X) \rightarrow h_{n}(|\mathbf{X}|)$ is in general not injective which contradicts a claim made in [1, Lemma 5.4(2)] in order to prove that Steenrod homology theories are uniquely determined by their restriction to ANR objects. In this context see also [13, Remark 7.7] and [14, Remark 2.18].

Proposition 3.9. For any Steenrod homology theory $\mathfrak{h}$ on $\mathbf{C M}_{\mathbf{n e}}$ or $\mathbf{C M}_{\mathbf{0}}$ the natural transformation $\beta: \lim ^{1} h_{n+1}(\mathbf{X}) \rightarrow h_{n}(X)$ can be chosen as the unique filler making the following diagram commute:

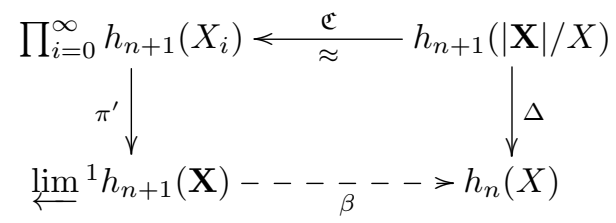


Here, $\mathfrak{C}$ is the obvious isomorphism induced by the cluster isomorphism (concerning $\Delta$ see Appendix B). Hence there is a commutative diagram

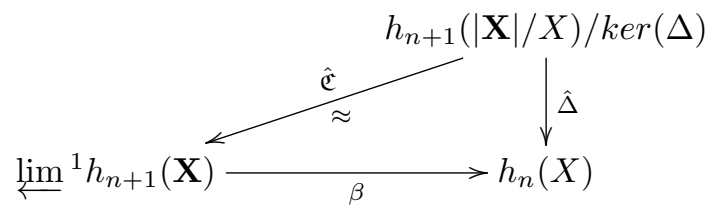

with an isomorphism $\hat{\mathfrak{C}}$ which is natural on $\mathbf{s e q}-\mathbf{C M}_{\mathbf{n e}}$ resp. seq- $\mathbf{C M}_{\mathbf{0}}$.

Proof. Can safely be left to the reader.

Recall that a homology theory $\mathfrak{h}$ is called continuous if the natural homomorphism $\mathbf{p}_{\#}: h_{n}(X) \rightarrow \lim h_{n}(\mathbf{X})$ is an isomorphism for all $n \in \mathbb{Z}$ and all $\mathbf{X} \in$ tow-C. We emphasize that we require $\mathfrak{h}$ to satisfy the exactness axiom, although continuity and exactness hold potential for conflict; e.g. ordinary Cech homology is exact only for a certain class of coefficient groups (including all algebraically compact groups). It is obvious that a maximally partially continuous homology theory is continuous if and only if $\lim ^{1} h_{n}(\mathbf{X})$ vanishes for all $\mathbf{X} \in$ tow-C.

Corollary 3.10. Each continuous homology theory is maximally partially continuous. In particular, $\lim ^{1} h_{n}(\mathbf{X})$ vanishes for all $\mathbf{X} \in$ tow-C.

Proof. By Remark 3.3 we see that each continuous homology theory is a Steenrod homology theory (take $\mathfrak{F}_{n}=0$ ). This implies maximal partial continuity.

Milnor's axiomatic characterization of ordinary Steenrod homology uses the cluster axiom for arbitrary sequences of pointed compacta. Inspection of Milnor's proof shows that it suffices to use the ANR cluster axiom, i.e. the cluster axiom for arbitrary sequences of pointed compact ANRs.

It is then easy to see that homology theories satisfying the strong excision axiom and the ANR cluster axiom are the same as ANR-partially continuous homology theories. This variant of our main theorem is the straight extension of [20, Corollary 1.5] from ordinary to generalized homology theories.

\section{Strong shape invariant homology theories}

As already pointed out, homology theories of strong type are known to be strong shape invariant and Steenrod homology theories are known to be Holsztynski shape invariant which at first glance seems to be more than strong shape invariant. But is it really more? This question could be answered by a brute "no" if it were true that each shape equivalence is a strong shape equivalence. However, this problem is still open (although there exists partial results, e.g. for finite-dimensional compacta). Irrespective of a final solution we have the following result.

Proposition 4.1. A homology theory is strong shape invariant if and only if it is Holsztynski shape invariant.

Proof. We show that a homology theory which inverts strong shape equivalences also inverts shape equivalences. Let us first consider the case of a reduced pointed 
homology theory on $\mathbf{C M}_{\mathbf{0}}$. An immediate offspring of [6, Corollary 3.3] is this:

(1) If $f: S\left(X, x_{0}\right) \rightarrow S\left(Y, y_{0}\right)$ is a pointed shape equivalence, then $f$ is a pointed strong shape equivalence.

Since suspensions of pointed shape equivalences are pointed shape equivalences and hence pointed strong shape equivalences, the suspension isomorphism $\sigma: h_{n}\left(X, x_{0}\right) \rightarrow$ $\left.h_{n+1}\left(S\left(X, x_{0}\right)\right)\right)$ proves the result.

Let us now consider unpointed homology. We show

(2) If $X$ and $Y$ are connected compacta and $f: S X \rightarrow S Y$ is a shape equivalence, then $f$ is a strong shape equivalence.

Choose a basepoint $\xi_{0} \in S X$ and let $\eta_{0}=f\left(\xi_{0}\right) \in S Y$. SY is path connected and representable as the inverse limit of simply connected compact polyhedra. Hence pro- $\pi_{1}\left(S Y, \eta_{0}\right)=0$ so that $S Y$ is pointed 1-movable. Hence $f:\left(S X, \xi_{0}\right) \rightarrow\left(S Y, \eta_{0}\right)$ is a pointed shape equivalence by $\left[\mathbf{7}\right.$, Theorem 5.1]. We have $\xi_{0}=\left[x_{0}, t_{0}\right]$ and $\eta_{0}=\left[y_{0}, t_{0}^{\prime}\right]$ with $x_{0} \in X$ and $y_{0} \in Y$. Let $p_{X}:\left(S X, \xi_{0}\right) \rightarrow S\left(X, x_{0}\right)$ and $p_{Y}:\left(S Y, \eta_{0}\right) \rightarrow S\left(Y, y_{0}\right)$ be the canonical quotient maps collapsing the segments through $x_{0}$ resp. $y_{0}$ to points. These maps are pointed strong shape equivalences (cf. [9, Corollaries 2, 3] or [17, Corollaries 10.7, 10.8]). Letting $s$ denote the pointed strong shape functor, we see that the pointed strong shape morphism $\phi=s\left(p_{Y}\right) \circ s(f) \circ s\left(p_{X}\right)^{-1}: S\left(X, x_{0}\right) \rightarrow S\left(Y, y_{0}\right)$ induces an isomorphism in the pointed shape category, hence by [6, Corollary 3.3] is an isomorphism in the pointed strong shape category. This implies that $f:\left(S X, \xi_{0}\right) \rightarrow$ $\left(S Y, \eta_{0}\right)$ is a pointed strong shape equivalence so that $f: S X \rightarrow S Y$ is a strong shape equivalence.

Now (2) applies to show that double suspensions of shape equivalences are strong shape equivalences, and we are done again.

\section{Appendix A. The first derived limit as a functor on tow-Ab}

For any category $\mathbf{D}$ let seq-D denote the following category of inverse sequences over $\mathbf{D}$. The morphisms $\mathbf{f}: \mathbf{X} \rightarrow \mathbf{Y}$ in seq-D are the systems $\mathbf{f}=\left(\varphi, f_{i}\right)$ where $\varphi: \mathbb{N} \rightarrow$ $\mathbb{N}$ is strictly increasing and $f_{i}: X_{\varphi(i)} \rightarrow Y_{i}$ are morphisms in $\mathbf{D}$ such that $f_{i} p_{\varphi(i)}^{\varphi(i+1)}=$ $q_{i} f_{\varphi(i+1)}$ for all $i \in \mathbb{N}$. A morphism $\mathbf{f}=\left(\varphi, f_{i}\right)$ is called a level morphism if $\varphi=i d$. The level morphisms form a subcategory $\mathbf{D}^{\mathbb{N}}$ of seq-D. The category tow-D of towers over $\mathbf{D}$ has as morphisms equivalence classes of morphisms in seq-D, where $\mathbf{f}=$ $\left(\varphi, f_{i}\right), \mathbf{f}^{\prime}=\left(\varphi^{\prime}, f_{i}^{\prime}\right): \mathbf{X} \rightarrow \mathbf{Y}$ are equivalent if there exists a strictly increasing function $\psi \geqslant \varphi, \varphi^{\prime}$ such that $f_{i} p_{\varphi(i)}^{\psi(i)}=f_{i}^{\prime} p_{\varphi^{\prime}(i)}^{\psi(i)}$ for all $i \in \mathbb{N}$. tow-D can be identified with a full subcategory of the pro-category pro-D whose objects are all inverse systems over $\mathrm{D}$ indexed by cofiltered small categories. See $[\mathbf{8}, \mathbf{1 7}, \mathbf{2 4}]$.

For an inverse sequence $\mathbf{G}=\left(G_{i}, \gamma_{i}\right)$ of abelian groups the first derived limit $\lim ^{1} \mathbf{G}$ occurs in form of the well-known definition

$$
\lim ^{1} \mathbf{G}=\operatorname{coker}\left(d_{\mathbf{G}}\right) \text { with } d_{\mathbf{G}}: \prod_{i=1}^{\infty} G_{i} \rightarrow \prod_{i=1}^{\infty} G_{i}, d_{\mathbf{G}}(g)_{i}=g_{i}-\gamma\left(g_{i+1}\right) .
$$

It is clear that $\lim ^{1}$ is a functor on $\mathbf{A} \mathbf{b}^{\mathbb{N}}$. We are in need of a pro-extension $\lim ^{1}$ : tow- $\mathbf{A b} \rightarrow \mathbf{A b}$ making the following diagram commute: 


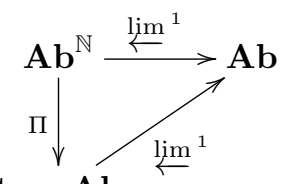

tow-Ab

The existence of such a pro-extension is well-known; it occurs as the first right derived functor of $\lim _{\text {: tow- }} \mathbf{A b} \rightarrow \mathbf{A b}$. In $[\mathbf{2 4}]$ this is concretized by showing that

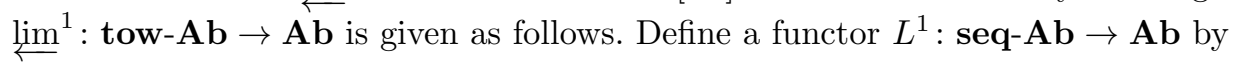

$$
L^{1}(\mathbf{G})=\prod_{i=1}^{\infty} G_{i}, L^{1}(\mathbf{f}): \prod_{i=1}^{\infty} G_{i} \rightarrow \prod_{i=1}^{\infty} H_{i}, L^{1}(\mathbf{f})(g)_{i}=\sum_{j=\varphi(i)}^{\varphi(i+1)-1} f_{i}\left(\gamma_{\varphi(i)}^{j}\left(g_{j}\right)\right),
$$

where $\mathbf{f}=\left(\varphi, f_{i}\right): \mathbf{G} \rightarrow \mathbf{H}$. Then

$$
\lim ^{1}([\mathbf{f}])([g])=\left[L^{1}(\mathbf{f})(g)\right] .
$$

\section{Appendix B. Homology theories of strong type}

We recall some useful facts from $[\mathbf{2 3}]$ about homology theories of strong type.

1. To each homology theory of strong type $\mathfrak{h}=\left(h_{n}, \sigma_{n}\right)$ on $\mathbf{C}=\mathbf{C M}_{\mathbf{n e}}, \mathbf{C M}_{\mathbf{0}}$ one can associate natural transformations $\Delta_{n}: h_{n+1}(X / A) \rightarrow h_{n}(A)$ such that there is a long exact sequence

$$
\cdots \stackrel{\Delta_{n}}{\rightarrow} h_{n}(A) \stackrel{i_{*}}{\rightarrow} h_{n}(X) \stackrel{p_{*}}{\rightarrow} h_{n}(X / A) \stackrel{\Delta_{n}-1}{\rightarrow} h_{n-1}(A) \stackrel{i_{*}}{\rightarrow} \cdots .
$$

This association between natural isomorphisms $\sigma_{n}$ and natural transformations $\Delta_{n}$ with the above property is $1-1$.

2. To each homology theory of strong type $\mathfrak{h}=\left(h_{n}, \sigma_{n}\right)$ on $\mathbf{C}=\mathbf{C M}_{\mathbf{n e}}, \mathbf{C M}_{\mathbf{0}}$ one can associate a homology theory of strong type $\operatorname{unred}(\mathfrak{h})=\left(\hat{h}_{n}, \partial_{n}\right)$ on $\mathbf{C M}^{\mathbf{2}}$ such that

$$
\hat{h}_{n}(X, A)=h_{n}(X / A) .
$$

In particular, we have on $\mathbf{C M} \subset \mathbf{C M}^{\mathbf{2}}$

$$
\hat{h}_{n}(X)=h_{n}\left(X^{+}\right) .
$$

$X / A$ and $X^{+}$are regarded as a pointed spaces in the canonical way for $\mathbf{C}=$ $\mathbf{C M}_{\mathbf{0}}$. Similarly we have on $\mathbf{C M}_{\mathbf{0}} \subset \mathbf{C M}^{\mathbf{2}}$

$$
\begin{gathered}
\hat{h}_{n}\left(X, x_{0}\right)=h_{n}(X) \text { for } \mathbf{C}=\mathbf{C M}_{\mathbf{n e}}, \\
\hat{h}_{n}\left(X, x_{0}\right)=h_{n}\left(X, x_{0}\right) \text { for } \mathbf{C}=\mathbf{C M}_{\mathbf{0}} .
\end{gathered}
$$

3. To each homology theory of strong type $\mathfrak{h}=\left(h_{n}, \partial_{n}\right)$ on $\mathbf{C M}^{\mathbf{2}}$ one can associate a homology theory of strong type $\operatorname{red}(\mathfrak{h})=\left(h_{n}^{\prime}, \sigma_{n}^{\prime}\right)$ on $\mathbf{C M}_{\text {ne }}$ resp. $\mathbf{C M}_{\mathbf{0}}$ such that

$$
\begin{gathered}
h_{n}^{\prime}(X)=k e r\left(c_{*}: h_{n}(X) \rightarrow h_{n}(*)\right) \text { on } \mathbf{C M}_{\mathbf{n e}} \\
h_{n}^{\prime}\left(X, x_{0}\right)=h_{n}\left(X,\left\{x_{0}\right\}\right) \text { on } \mathbf{C M}_{\mathbf{0}}
\end{gathered}
$$


4. The above transformation procedures are inverse to each other in the sense that $\operatorname{red}(\operatorname{unred}(\mathfrak{h}))$ and $\operatorname{unred}(\operatorname{red}(\mathfrak{h}))$ are naturally isomorphic to the original $\mathfrak{h}$. In that sense the three kinds of homology theories of strong type living on $\mathbf{C M}_{\mathbf{n e}}, \mathbf{C M}_{\mathbf{0}}, \mathbf{C M}^{\mathbf{2}}$ are equivalent.

Definition B.1. A triple of homology theories of strong type $\left(\mathfrak{h}^{(n e)}, \mathfrak{h}^{(0)}, \mathfrak{h}\right)$ living on $\mathbf{C M}_{\mathbf{n e}}, \mathbf{C M}_{\mathbf{0}}, \mathbf{C M}^{2}$, respectively, is called an associated triple if $\mathbf{u n r e d}\left(\mathfrak{h}^{(n e)}\right)$ and $\operatorname{unred}\left(\mathfrak{h}^{(0)}\right)$ are naturally isomorphic to $\mathfrak{h}$.

Each homology theory of strong type living on one of the categories $\mathbf{C M}_{\mathbf{n e}}, \mathbf{C M}_{\mathbf{0}}$, $\mathbf{C M}^{2}$ occurs in an associated triple which is up to natural isomorphism unique.

For homology theories which are not of strong type there are only partial analogues. Whereas homology theories on $\mathbf{C M}_{\mathbf{n e}}$ and $\mathbf{C M}^{\mathbf{2}}$ are equivalent (see Kelly [15]), this is not true for homology theories on $\mathbf{C M}_{\mathbf{0}}$ and $\mathbf{C M}^{\mathbf{2}}$.

\section{References}

[1] F.W. Bauer, Extensions of generalized homology theories, Pacific J. Math. 128 (1987), 25-61

[2] N.A. Berikashvili, The Steenrod-Sitnikov homology theory on the category of compact spaces, Dokl. Akad. Nauk SSSR 254 (1980), 1289-1291

[3] N.A. Berikashvili, Axiomatics of the Steenrod-Sitnikov homology theory on the category of compact Hausdorff spaces, Tr. Mat. Inst. Steklova 154 (1983), $24-37$

[4] N.A. Berikashvili, On the uniqueness of homology theory on the category of compact spaces, Proc. A. Razmadze Math. Inst. Georgian Acad. Sci. $\mathbf{8 3}$ (1986), 19-25

[5] A. Calder and H.M. Hastings, Realizing strong shape equivalences, J. Pure Appl. Algebra 20 (1981), 129-156

[6] F.W. Cathey, Strong shape theory, in: Shape Theory and Geometric Topology (S. Mardešić and J. Segal, eds.), Lecture Notes in Math. 870, Springer, Berlin, 1981, 215-238

[7] J. Dydak, Pointed and unpointed shape and pro-homotopy, Fund. Math. 107 (1980), 57-69

[8] D.A. Edwards and H.M. Hastings, Čech and Steenrod Homotopy Theories with Applications to Geometric Topology, Lecture Notes in Math. 542, Springer, Berlin, Heidelberg, New York, 1976

[9] B. Günther, Properties of normal embeddings concerning strong shape theory II, Tsukuba J. Math. 16 (1992), 429-438

[10] W. Holsztynski, An extension and axiomatic characterization of Borsuk's theory of shape, Fund. Math. 70 (1971), 57-68

[11] H.N. Inassaridze, On the Steenrod homology theory of compact spaces, Michigan Math. J. 38 (1991), 323-338

[12] H.N. Inassaridze and L.D. Mdzinarishvili, On the connection between continuity and exactness in homology theory, Bull. Georgian Acad. Sci. 99 (1980), $317-320$ 
[13] D.S. Kahn, J. Kaminker and C. Schochet, Generalized homology theories on compact metric spaces, Michigan Math. J. 24 (1977), 203-224

[14] J. Kaminker and C. Schochet, K-Theory and Steenrod homology: Applications to the Brown-Douglas-Fillmore Theory of operator algebras, Trans. Amer. Math. Soc. 227 (1977), 63-107

[15] G.M. Kelly, Single-space axioms for homology theory, Proc. Cambridge Philos. Soc. 55 (1959), 10-22

[16] J. Krasinkiewicz, On a method of constructing ANR-sets. An application of inverse limits, Fund. Math. 92 (1976), 95-112

[17] S. Mardešić, Strong Shape and Homology, Springer, Berlin, Heidelberg, New York, 2000

[18] L.D. Mdzinarishvili, Application of the shape theory in the characterization of exact homology theories and the strong shape homotopic theory, in: Shape Theory and Geometric Topology (Dubrovnik, 1981), Lecture Notes in Math. 870, Springer, Berlin, New York, 1981, 253-262

[19] L.D. Mdzinarishvili, Universelle Koeffizientenfolgen für den lim-Funktor und Anwendungen, Manuscripta Math. 48 (1984), 255-273

[20] L.D. Mdzinarishvili, On homology extensions, Glas. Mat. Ser. III 21(41) (1986), 455-482

[21] L.D. Mdzinarishvili, On exact homology, in: Geometric Topology and Shape Theory (Dubrovnik, 1987), Lecture Notes in Math. 1283, Springer, Berlin, New York, 1987, 164-182

[22] J. Milnor, On the Steenrod Homology Theory, mimeographed notes, Berkeley, 1960; also in: Novikov Conjectures, Index Theorems and Rigidity Vol. 1, (S.C. Ferry, A. Ranicki and J. Rosenberg, eds.), London Math. Soc. Lecture Note Ser. 226, Cambridge University Press, Cambridge, 1995, 79-96

[23] P. Mrozik, Generalized Steenrod homology theories are strong shape invariant, Homology Homotopy Appl. 12 (2010), 1-23

[24] P. Mrozik, Extending functors from the category of strict morphisms of inverse systems to the associated pro-category with applications to the first derived limit, Tsukuba J. Math. 41 (2017), 265-296.

Peter Mrozik pmrozik@gmx.de

Berliner Str. 11, 61194 Niddatal, Germany 